\title{
Search for invisible decays at BESIII
}

\section{X.D. Shi for the BESIII Collaboration ${ }^{a, b, *}$}

${ }^{a}$ State Key Laboratory of Particle Detection and Electronics, 96 Jinzhai Road, Hefei, China

${ }^{b}$ University of Science and Technology of China, 96 Jinzhai Road, Hefei, China

E-mail: xiaodong.shi@mail.ustc.edu.cn

BESIII has collected $(448.1 \pm 2.9) \times 10^{6} \psi(3686)$ and $(1.0087 \pm 0.0044) \times 10^{10} \mathrm{~J} / \psi$ data sample. We report recent searches of the $\mathrm{J} / \psi \rightarrow \gamma+$ invisible and $\Lambda \rightarrow$ invisible based on these huge data sets, where the second one is the first search of baryon's invisible decay. With no significant signal observed, the upper limits at $90 \%$ C.L. are calculated, which provide constraints on the corresponding new physics models.

*** The European Physical Society Conference on High Energy Physics (EPS-HEP2021), *** *** 26-30 July $2021 * * *$

*** Online conference, jointly organized by Universität Hamburg and the research center DESY ***

${ }^{*}$ Speaker 


\section{Introduction}

Despite the success of the Standard Model (SM), there are some experimental evidences related to New Physics. Dark matter is one of the most compelling one: they account for $\sim 27 \%$ of the total universe, but can't be explained by the SM. Although there are so many evidences in astronomy about dark matter, they haven't been observed directly yet. [1] One way to directly search for dark matter is searching for invisible decays at collider experiments.

BESIII [2] is an $e^{+} e^{-}$collider experiment running at $\tau$-charm energy region with peak luminosity at $10^{33} \mathrm{~cm}^{-2} \mathrm{~s}^{-1}$ located at the Beijing Electron Positron Collider (BEPCII) [3]. In the last more than 10 years running period, BESIII has accumulated huge data sample, including world largest $\mathrm{J} / \psi, \psi^{\prime}, \psi^{\prime \prime}$ data set by direct $e^{+} e^{-}$annihilation. [4] With these huge clean data samples, BESIII has performed several searches for invisible decays. [5-7] This report summaries one recent result and one preliminary result at BESIII related to search for invisible decay. [7, 8]

\section{Search for $\mathbf{J} / \psi \rightarrow \gamma+$ invisible}

The series of Supersymmetric Standard Models [9], including the Next-to-Minimal Supersymmetric Model (NMSSM) [10], predict a light CP-odd pseudoscalar Higgs boson $A^{0}$ and a series of neutralinos. The light stable neutralino $\left(\chi^{0}\right)$, which is a candidate of dark matter, can couple with Standard Model particles via the $A^{0}$ boson. The $A^{0}$ can be produced in the radiative decay of a quarkonium vector state, $V[11,12]$. The branching ratio of such a radiative decay is:

$$
\frac{\mathcal{B}\left(V \rightarrow \gamma A^{0}\right)}{\mathcal{B}\left(V \rightarrow \mu^{+} \mu^{-}\right)}=\frac{G_{F} m_{q}^{2} g_{q}^{2} C_{Q C D}}{\sqrt{2} \pi \alpha}\left(1-\frac{m_{A^{0}}^{2}}{m_{V}^{2}}\right),
$$

where $m_{A^{0}}, m_{V}$ and $m_{q}$ are the masses of the $A^{0}$, the quarkonium state, and the corresponding quark, respectively; $\alpha$ is the fine structure constant; $G_{F}$ is the Fermi coupling constant; $C_{Q C D}$ is the combined QCD radiative and relativistic corrections [13]; and $g_{q}$ is the Yukawa coupling of the $A^{0}$ field to the quark-pair, and for the charm quark is $g_{c}=\cos \theta_{\mathrm{A}} / \tan \beta$ and for the bottom quark is $g_{b}=\cos \theta_{\mathrm{A}} \tan \beta$, where $\tan \beta$ is the usual ratio of vacuum expectation values and $\theta_{A}$ is the Higgs mixing angle [14]. The $A^{0}$ may decay into two $\chi^{0}$ s, then is "invisible" to the detector.

Previously many collider experiments, such as CLEO-c [15], BaBar [16] and Belle [17], has searched for $\mathrm{J} / \psi$ or $\Upsilon(1 \mathrm{~S})$ radiative decays into invisible particles, but no signal was observed. The huge $\psi^{\prime}$ data set collected at BESIII provides another chance to search for $\mathrm{J} / \psi \rightarrow \gamma+$ invisible, through $\psi^{\prime} \rightarrow \pi^{+} \pi^{-} \mathrm{J} / \psi$ process.

Firstly, the $\mathrm{J} / \psi$ sample is tagged by reconstructing $\pi^{+} \pi^{-}$pairs. With several criteria on $\pi^{+} \pi^{-}$, the yield of selected $\mathrm{J} / \psi$ sample is evaluated by a fit on recoiling mass of $\pi^{+} \pi^{-}\left(M_{\pi^{+} \pi^{-}}^{r e c}\right.$, shown as Fig. 1 (a). To better describe the peak's shape, a control sample of $\psi^{\prime} \rightarrow \pi^{+} \pi^{-} \mathrm{J} / \psi, \mathrm{J} / \psi \rightarrow \mathrm{e}^{+} \mathrm{e}^{-}$ is selected. Then the signal shape is modeled using the $M_{\pi^{+} \pi^{-}}^{r e c}$ distribution of the control sample convoluted with a Gaussian function, which represents the resolution difference between $\mathrm{J} / \psi \rightarrow$ $\mathrm{e}^{+} \mathrm{e}^{-}$and the $\mathrm{J} / \psi$ inclusive decay. According to the fit, the $\mathrm{J} / \psi$ 's yield is determinated as $8.848 \times 10^{7}$ in the $\mathrm{J} / \psi$ 's signal region $\left([3.082,3.112] \mathrm{GeV} / c^{2}\right)$.

Then the signal process is searched based on the selected $\mathrm{J} / \psi$ sample. Events with only two charged pions in the $\mathrm{J} / \psi$ 's signal region and one good shower are selected. The good photon, 
identified as the signal shower, is required from the EMC Barrel region. The direction of the recoiled invisible particle is also required to be within the EMC Barrel region. With the above criteria, detailed MC studies indicate that the dominant background are from $\psi^{\prime} \rightarrow \pi^{+} \pi^{-} \mathrm{J} / \psi$ with $\mathrm{J} / \psi$ decays into final states including neutral hadrons, e.g., $n \bar{n}, \gamma K_{L} K_{L}$. To identify $\gamma$ from $n, \bar{n}, K_{L}$, the accessible shower shape variables are studied through the control samples of the decay processes $\mathrm{J} / \psi \rightarrow \pi^{+} \pi^{-} \pi^{0}\left(\pi^{0} \rightarrow \gamma \gamma\right), \mathrm{J} / \psi \rightarrow p \pi^{-} \bar{n}+$ c.c. and $\mathrm{J} / \psi \rightarrow K \pi K_{L}, \mathrm{~J} / \psi \rightarrow \pi^{+} \pi^{-} \phi\left(\phi \rightarrow K_{S} K_{L}\right)$. After optimization, a series of requirements on shower shape variables are applied. The selection efficiency and shower's energy of the neutral hadrons are also extracted from these control samples, since the MC simulation is not reliable.
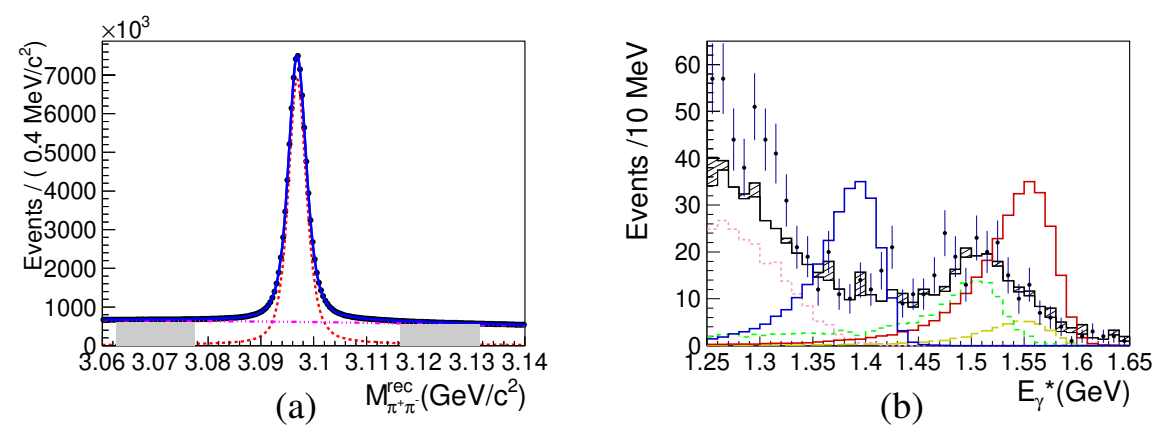

Figure 1: (a) Fit to the $M_{\pi^{+} \pi^{-}}^{r e c}$ distribution. The blue solid line is the sum of signal (red dashed line) and background (pink dashed line). The shaded region is the sideband region for non-J/ $\psi$ background study. (b) The $E_{\gamma}^{*}$ distribution. Data is shown with black dots. The total background from $\psi(3686) \rightarrow \pi^{+} \pi^{-} \mathrm{J} / \psi$, estimated from MC simulation, is shown with the black solid line. The dominant backgrounds are shown as three dashed lines. and includes contributions from the subsequent decays $\mathrm{J} / \psi \rightarrow \gamma \pi^{0}$ (long dashed yellow line), The red and blue solid lines show the signal shape with 0 and $1 \mathrm{GeV} / c^{2}$ mass assumptions, respectively.

The signal shower's energy in the $\mathrm{J} / \psi$ rest frame, $E_{\gamma}^{*}$, is used to identify the signal. Shown in Fig. 1 (b), the dominant backgrounds are from $\psi(3686) \rightarrow \pi^{+} \pi^{-} \mathrm{J} / \psi$ with subsequent decays $\mathrm{J} / \psi \rightarrow \gamma \mathrm{K}_{\mathrm{L}} \mathrm{K}_{\mathrm{L}}, \gamma \eta$ and $\gamma \pi^{0}$, where the latter two are peak backgrounds. A series of unbinned likelihood fits on the $E_{\gamma}^{*}$ are preformed, with different invisible particle's mass assumption from 0 up to $1.2 \mathrm{GeV} / c^{2}$. In the fits, the signal shape is taken from the signal MC simulation convoluted with a Gaussian function representing the resolution difference between data and MC. The background shape is described by the sum of an exponential function and two crystal ball functions with fixed amplitudes and shapes presenting for the background of $\psi(3686) \rightarrow \pi^{+} \pi^{-} \mathrm{J} / \psi$ with subsequent decay $\mathrm{J} / \psi \rightarrow \gamma \eta$ and $\gamma \pi^{0}$, respectively, where amplitudes and shapes are estimated by the exclusive MC simulation. As no strong peaks are observed in all fits, the upper limits at $90 \%$ confidence level are calculated taking into account all systematic uncertainties by using the modified frequentist method known as $C L_{s}[19,20]$ combined with the asymptotic approximation [21]. The results are shown in Fig. 2 (a), where the data is consistent with the zero-signal assumption in the $2 \sigma$ region. And for the zero mass assumption of the invisible particle the upper limit is $7.0 \times 10^{-7}$.

To further investigate the physical parameters in NMSSM, according to Ref. [12] and Eq. (1), the upper limits of $g_{c} \times \tan ^{2} \beta \times \sqrt{\mathcal{B}\left(A^{0} \rightarrow \text { invisible }\right)}$ based on the measured upper limits of this work are extracted for $\tan \beta=0.6,0.7$ and 0.8, individually, as presented in Fig. 2 (b). The extracted 
results are directly compared to $g_{b} \times \sqrt{\mathcal{B}\left(A^{0} \rightarrow \text { invisible }\right)}\left(=g_{c} \times \tan ^{2} \beta \times \sqrt{\mathcal{B}\left(A^{0} \rightarrow \text { invisible }\right)}\right)$, which is obtained based on the Belle results [17] and also presented in Fig. 2 (b). For $\tan \beta \leq 0.6$, our result's sensitivity is better than Belle's. Combining the results from Belle [17], we also extract upper limits on $\cos \theta_{A}\left(=\sqrt{g_{b} g_{c}}\right) \times \sqrt{\mathcal{B}\left(A^{0} \rightarrow \text { invisible }\right)}$, as presented in Fig. 2 (c).

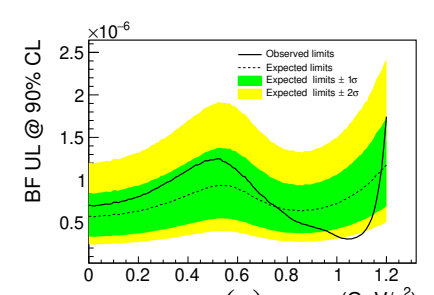

(a) $m_{\text {invisible }}\left(G e V / c^{2}\right)$

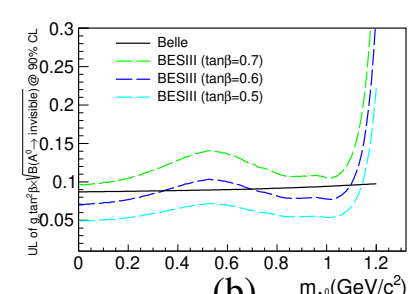

(b) $\quad m_{A^{0}}\left(G e V / c^{2}\right)$

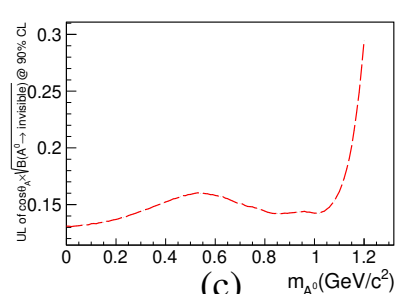

(c)

Figure 2: Upper limits at the $90 \%$ C.L. for (a) Branching ratio of $\mathrm{J} / \psi \rightarrow \gamma+$ invisible, (b) $g_{c} \times \tan ^{2} \beta\left(g_{b}\right) \times$ $\sqrt{\mathcal{B}\left(\mathrm{A}^{0} \rightarrow \text { invisible }\right)}$, and (c) $\sqrt{g_{b} g_{c}} \times \sqrt{\mathcal{B}\left(A^{0} \rightarrow \text { invisible }\right)}$.

\section{Search for $\Lambda \rightarrow$ invisible}

The asymmetry between matter and anti-matter in the universe indicate that the baryon number (B) shall be violated. [22] Noticing the baryon matter density and the dark matter density are similar, $\rho_{\mathrm{DM}} \approx 5.4 \rho_{\text {baryon }}$, some models try to understand dark matter and baryon asymmetry simultaneously.[23-25] Through a neutron portal operator $u^{c} d^{c} d^{c}$ [26, 27], where $q^{c}$ is the righthanded quark, these models import the couple between dark sector and SM sector, which can generally introduce $B$ violation to the SM sector. The discrepancy of measured $\tau(\mathrm{n})$ between the bottle method and the beam method [28-30] can also be explained by these models, by assuming $1 \%$ of the neutron decays into dark matter particles [31]. Moreover, allowing for quarks from the 2nd and 3rd generation in the neutron portal will generally result in new portals, which can lead to heavy flavor meson and baryon decays into dark sector particles. [32] Specifically, the $\Lambda$ baryon can decay into dark matter with these models.

The huge $\mathrm{J} / \psi$ data $\left((1.0087 \pm 0.0044) \times 10^{10}\right)$ accumulated at BESIII provide an excellent chance to search for the $\Lambda$ baryon's invisible decay through the $\mathrm{J} / \psi \rightarrow \Lambda \bar{\Lambda}$ process, where the Lam $\bar{m} d a$ is tagged by $\bar{p} \pi^{+}$. This search is also the first invisible decay search in baryon sector.

Firstly, the $\bar{\Lambda}$ is reconstructed by $\bar{p} \pi^{+}$. Specially, to guarantee all the showers are assembled in the corresponding event, at least one charged tracks shall leave cluster information in the TOF. A maximum likelihood fit is performed on the recoil mass distribution $R M\left(\bar{p} \pi^{+}\right)$, to extract the yield of $\bar{\Lambda}$. As the Fig. 3 (a) shown, there is almost no background, and the $N(\Lambda)$ is determined as $4154428 \pm 2040$.

The signal process, $\Lambda \rightarrow$ invisible, is searched from the previous selected sample. Besides the $\bar{p}$ and $\pi^{+}$, there should be no extra charged track. Then the sum energy of total showers in EMC except those associated with any charged tracks, $E_{\mathrm{EMC}}$, is used to identify the signal. It is found that for the main background, $\mathrm{J} / \psi \rightarrow \Lambda \bar{\Lambda}, \bar{\Lambda} \rightarrow \overline{\mathrm{p}} \pi^{+}, \Lambda \rightarrow \mathrm{n} \pi^{0}$ process, the MC simulation of the $E_{\mathrm{EMC}}$ is not accurate, especially for the showers from n. To well model the background's $E_{\mathrm{EMC}}$, a control sample $\mathrm{J} / \psi \rightarrow \Lambda \bar{\Lambda}, \bar{\Lambda} \rightarrow \overline{\mathrm{p}} \pi^{+}, \Lambda \rightarrow \mathrm{n} \pi^{0}$ is selected and used to correct the showers' energy 
of $\mathrm{n}$ in the background $\mathrm{MC}$. The $E_{\mathrm{EMC}}$ distributions of data, background $\mathrm{MC}$ after correction, and signal MC are shown in Fig. 3 (b). A fit is performed on this distribution, and no significant signal is found. By using the modified frequentist method [19, 20], the upper limit at $90 \%$ confidence level is calculated as $7.4 \times 10^{-5}$ including the systematic uncertainty.
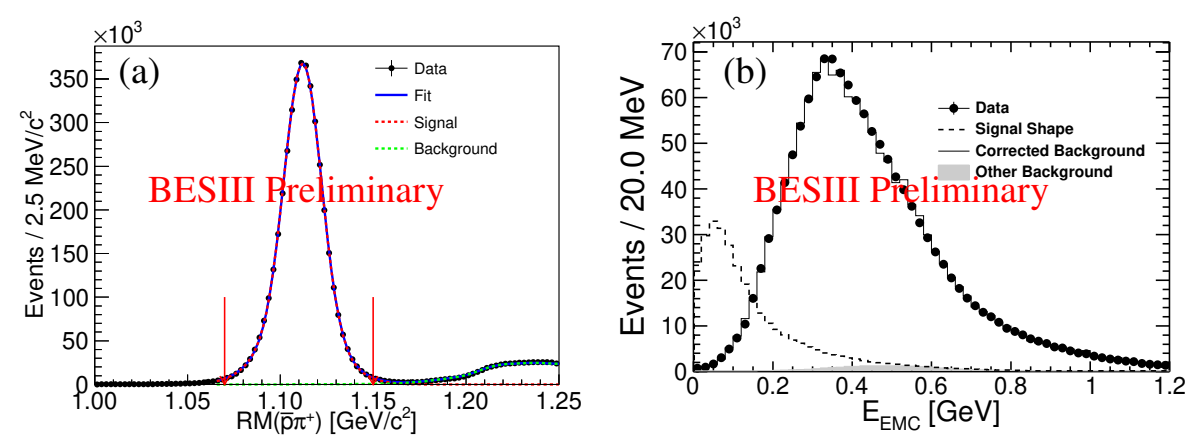

Figure 3: (a) Fit to the $R M\left(\bar{p} \pi^{+}\right)$distribution. (b) The $E_{\mathrm{EMC}}$ distribution.

\section{Summary}

With the huge data sets collected at BESIII, several searches for invisible decays are performed. In this talk, two recent works are reported. Using $(448.1 \pm 2.9) \times 10^{6} \psi(3686)$ decays collected at BESIII, the $\mathrm{J} / \psi \rightarrow \gamma+$ invisible process is searched. With no significant signal found, the upper limits at $90 \%$ confidence level are calculated for different $m_{\text {invisible }}$ assumptions up to $1.2 \mathrm{GeV} / c^{2}$. [7] Using $(1.0087 \pm 0.0044) \times 10^{10} \mathrm{~J} / \psi$ decays collected at BESIII, the $\Lambda \rightarrow$ invisible process is searched. With no significant signal found, the upper limit at $90 \%$ confidence level is calculated as $7.4 \times 10^{-5}$. [8]

\section{References}

[1] G. Bertone, D. Hooper and J. Silk, Phys. Rept. 405, 279 (2005)

[2] M. Ablikim et al. [BESIII Collaboration], Nucl. Instrum. Meth. A 614, 345 (2010); C. Z. Yuan and S. L. Olsen, Nature Rev. Phys. 1 (2019) no.8, 480.

[3] C. H. Yu et al., Proceedings of IPAC2016, Busan, Korea, 2016, doi:10.18429/JACoWIPAC2016-TUYA01.

[4] M. Ablikim et al. [BESIII], Chin. Phys. C 44 (2020) no.4, 040001

[5] M. Ablikim et al. [BESIII], Phys. Rev. D 87 (2013) no.1, 012009

[6] M. Ablikim et al. [BESIII], Phys. Rev. D 98 (2018) no.3, 032001

[7] M. Ablikim et al. [BESIII], Phys. Rev. D 101 (2020) no.11, 112005

[8] M. Ablikim et al. [BESIII], [arXiv:2110.06759 [hep-ex]]. 
[9] P. Fayet, Phys. Lett. 69B, 489 (1977).

[10] U. Ellwanger, C. Hugonie and A. M. Teixeira, Phys. Rept. 496, 1 (2010).

[11] F. Wilczek, Phys. Rev. Lett. 39, 1304 (1977).

[12] P. Fayet, Phys. Rev. D 75, 115017 (2007).

[13] P. Nason, Phys. Lett. B 175, 223 (1986).

[14] J. F. Gunion, D. Hooper and B. McElrath, Phys. Rev. D 73, 015011 (2006).

[15] J. Insler et al. [CLEO Collaboration], Phys. Rev. D 81, 091101 (2010).

[16] P. del Amo Sanchez et al. [BaBar Collaboration], Phys. Rev. Lett. 107, 021804 (2011).

[17] I. S. Seong et al. [Belle Collaboration], Phys. Rev. Lett. 122, 011801 (2019).

[18] D. N. Gao, Phys. Rev. D 90, 077501 (2014).

[19] A. L. Read, J. Phys. G 28, 2693 (2002).

[20] A. L. Read, CERN-OPEN-2000-205.

[21] G. Cowan, K. Cranmer, E. Gross and O. Vitells, Eur. Phys. J. C 71, 1554 (2011); Erratum: [Eur. Phys. J. C 73, 2501 (2013)].

[22] A. D. Sakharov, Pisma Zh. Eksp. Teor. Fiz. 5, 32 (1967) [JETP Lett. 5, 24 (1967)] [Sov. Phys. Usp. 34, 392 (1991)] [Usp. Fiz. Nauk 161, 61 (1991)].

[23] J. Shelton and K. M. Zurek, Phys. Rev. D 82, 123512 (2010).

[24] H. Davoudiasl, D. E. Morrissey, K. Sigurdson and S. Tulin, Phys. Rev. Lett. 105, 211304 (2010).

[25] P. H. Gu, M. Lindner, U. Sarkar and X. Zhang, Phys. Rev. D 83, 055008 (2011).

[26] K. Petraki and R. R. Volkas, Int. J. Mod. Phys. A 28, 1330028 (2013).

[27] K. M. Zurek, Phys. Rept. 537, 91 (2014).

[28] S. Arzumanov, L. Bondarenko, S. Chernyavsky, P. Geltenbort, V. Morozov, V. V. Nesvizhevsky, Y. Panin and A. Strepetov, Phys. Lett. B 745 (2015), 79-89

[29] A. T. Yue, M. S. Dewey, D. M. Gilliam, G. L. Greene, A. B. Laptev, J. S. Nico, W. M. Snow and F. E. Wietfeldt, Phys. Rev. Lett. 111 (2013) no.22, 222501

[30] N. Sumi, K. Hirota, G. Ichikawa, T. Ino, Y. Iwashita, S. Kajiwara, Y. Kato, M. Kitaguchi, K. Mishima and K. Morikawa, et al. JPS Conf. Proc. 33 (2021), 011056

[31] B. Fornal and B. Grinstein, Phys. Rev. Lett. 120, 191801 (2018) [erratum: Phys. Rev. Lett. 124, 219901 (2020)].

[32] G. Elor, M. Escudero and A. Nelson, Phys. Rev. D 99 (2019) no.3, 035031 